\title{
SÍNDROME DA CABEÇA CAÍDA EM DOENÇA DO NEURÔNIO MOTOR
}

\author{
Paulo José Lorenzoni', Marcos Christiano Lange ${ }^{1}$, Cláudia S.K. Kay', \\ Luiz G.M.P. de Almeida², Hélio A.G. Teive3, Rosana H. Scola4, Lineu C. Werneck ${ }^{5}$
}

\begin{abstract}
RESUMO - A síndrome da cabeça caída é causada por diminuição de força nos músculos extensores do pescoço sendo encontrada em diversas doenças neuromusculares, bem como, na esclerose lateral amiotrófica. Descrevemos o caso de três mulheres com diagnóstico de doença do neurônio motor com quadro clínico de disfagia e diminuição de força em músculos cervicais que evoluiu com queda da cabeça. A investigação mostrou ressonância magnética de crânio e coluna cervical normais; e a eletromiografia de agulha com desinervação ativa e crônica em músculos bulbares e dos segmentos cervical, torácico e lombo-sacro. Discutimos as características da doença, especialmente suas manifestações clínicas e os achados eletro n euromiográficos, dando ênfase à necessidade de investigação da cabeça caída na suspeita de doença do neurônio motor.
\end{abstract}

PALAVRAS-CHAVE: síndrome da cabeça caída, esclerose lateral amiotrófica, doença do neurônio motor inferior, eletromiografia.

\section{Dropped head syndrome in motor neuron disease}

ABSTRACT - Dropped head is a syndrome caused by weakness of the neck extensor muscles found in different kinds of neuromuscular disorders and also in amyotrophic lateral sclerosis. This is a cases report of three women with motor neuron disease with beginning of dysphagia and cervical weakness that it evolved with dropped head. The investigation showed normal magnetic resonance imaging of brain and cervical column. Needle electromyography showed active and chronic denervation in bulbar muscles and cervical, thoracic and lumbosacral segments. We discuss the characteristic of disease, specially the clinical manifestations and electromyography features, with emphasis at the clinical evaluation of dropped head in the suspicion of motor neuron disease.

KEY WORDS: dropped head syndrome, amyotrophic lateral sclerosis, lower motor neuron disease, electromyography.

A síndrome da cabeça caída (do inglês "dropped head") caracteriza-se por fraqueza da musculatura extensora do pescoço, levando os pacientes a apresenta remo típico aspecto da cabeça flexionada com o queixo em contato com a parede torácica, limitando suas atividades de vida, diárias e sociais ${ }^{1-3}$.

Diferentes etiologias estão relacionadas com a síndrome da cabeça caída (SCC), incluindo miopatias, doenças da junção neuromuscular, neuropatias, doenças do sistema nervoso central e doenças metabólicas ${ }^{2-10}$. Existem ainda pacientes sem etiologia definida para a cabeça caída, sendo observado em alguns uma miopatia cervical extensora isolada (isolated neck extensor myopathy), a qual também é reconhecida como miopatia axial ou d ropped head syndro - $m e^{11-13}$. A presença de SCC é raramente descrita em pacientes port a $d$ o res de doença do neurônio motor (DNM) como a esclerose lateral amiotrófica (ELA). O seu mecanismo fisiopatológico ainda não esta bem escla recido, sendo uma hipótese o envolvimento das células localizadas no corno anterior da medula que inervam a musculatura paraespinhal 1,2,12,14.

Neste artigo, descrevemos três pacientes com SCC associada a DNM.

\section{CASOS}

Caso 1 - Mulher de 60 anos que iniciou quadro de disfagia e diminuição de força muscular em membros superiores (MS). Após um mês do início dos sintomas evoluiu com diminuição da força muscular em região cervical apre-

Se rviço de Neurologia e Doenças Neuromusculares do Hospital de Clínicas da Universidade Federal do Paraná, Curitiba PR, Brasil (UFPR): 'Médico Neurologista' ${ }^{2}$ Acadêmico de Medicina; ${ }^{3}$ Professor Assistente; ${ }^{4}$ Professor Adjunto; ${ }^{5}$ Professor Titular.

Recebido 29 Abril 2005, recebido na forma final 1 Agosto 2005. Aceito 26 Setembro 2005.

Dra. Rosana Herminia Scola- Serviço de Doenças Neuro musculares / Hospital de Clínicas da UFPR - Rua General Carneiro 181 / $3^{\circ}$ andar -80060-900 Curitiba PR-Brasil. E-mail: scola@hc.ufpr.br 
Tabela 1. Eletromiografia de agulha dos músculos do segmento bulbar e cervical.

\begin{tabular}{|c|c|c|c|c|c|c|c|c|c|c|c|c|c|c|c|}
\hline \multirow{2}{*}{$\begin{array}{l}\text { Músculos } \\
\text { Casos }\end{array}$} & \multicolumn{3}{|c|}{ Fibrilação } & \multicolumn{3}{|c|}{ Fasciculação } & \multicolumn{3}{|c|}{ Duração } & \multicolumn{3}{|c|}{ Polifasia } & \multicolumn{3}{|c|}{ Recrutamento } \\
\hline & 1 & 2 & 3 & 1 & 2 & 3 & 1 & 2 & 3 & 1 & 2 & 3 & 1 & 2 & 3 \\
\hline Língua & +++ & +++ & + & 0 & ++ & + & $A$ & $A$ & NR & + & + & NR & $\mathrm{D}$ & D & NR \\
\hline Paraespinhais cervicais & +++ & + & 0 & 0 & + & 0 & A & $A$ & NR & + & + & NR & $\mathrm{D}$ & D & NR \\
\hline Trapézio & ++ & 0 & NR & 0 & 0 & NR & A & $A$ & NR & +++ & + & NR & $\mathrm{D}$ & D & NR \\
\hline Esternocleidomastoideo & ++ & 0 & 0 & 0 & + & 0 & A & * & $\mathrm{N}$ & ++ & * & 0 & $\mathrm{D}$ & * & $\mathrm{N}$ \\
\hline Escalenos & 0 & + & NR & 0 & 0 & NR & A & $\mathrm{N}$ & NR & ++ & 0 & NR & $D$ & D & NR \\
\hline
\end{tabular}

+, raras; ++, ocasionais; +++, freqüentes; 0, ausente; NR, não realizado, *, ausência de atividade motora voluntária; N, normal; D, diminuído; $\mathrm{A}$ aumentado.

sentando queda de cabeça. O exame neurológico mostrava disartria, fasciculações em língua e MS. O exame dos MS revelou trofismo muscular diminuído e tônus muscular disc retamente aumentado. A diminuição de força muscular era grau 3 nos MS e de grau 0 nos músculos extensores cervicais, segundo critérios do Medical Research Council (MRC). O exame ainda revelou sinais de liberação piramidal e as sensibilidades tátil, vibrató ria e dolorosa estavam preservadas. $O$ restante do exame neurológico estava normal. $A$ e le tromiografia de agulha mostrou sinais de desinervação crônica e ativa com fibrilações e fasciculações em músculos dos segmentos bulbar, cervical e torácico. Iniciou-se tratamento com riluzole $100 \mathrm{mg} / \mathrm{dia}$, porém não houve melhora do quadro e a paciente evoluiu para o óbito após dois anos do início da doença.

Caso 2 - Mulher de 66 anos que iniciou quadro de disfagia, disfonia e diminuição de força muscular em membro superior e inferior esquerdos. Após um ano do início dos sintomas evoluiu com diminuição da força muscular em região cervical com dificuldade para sustentar a cabeça (Figura). O exame neurológico mostrava disfonia, fasciculações em língua, tônus muscular difusamente aumentado, força muscular diminuída em membro superior e inferior esquerdos grau 4 e músculos extensores cervicais grau 1 (MRC) e sinais de liberação piramidal. As sensibilidades tátil, vibratória e dolorosa estavam preservadas. A eletromio- grafia de agulha mostrou sinais de desinervação crônica e ativa com fibrilações e fasciculações em músculos dos segmentos bulbar, cervical, lombar e sacral. Há três anos iniciou tratamento com riluzole $100 \mathrm{mg} / \mathrm{dia}$ sem melhora do quadro.

Caso 3 - Mulher de 54 anos que iniciou quadro de disfagia e disartria. Após dois anos do início dos sintomas apresentou dificuldade para sustentar a cabeça devido diminuição da força muscular em região cervical evoluindo com queda da cabeça. $\mathrm{O}$ exame neurológico mostrava anartria, fasciculações em língua, redução do reflexo do vômito, paresia bilateral da elevação do palato e força muscular diminuída em músculos extensores cervicais grau 1 (MRC) e o restante do exame neurológico foi normal. A eletromiografia de agulha mostrou sinais de desinervação ativa com fibrilações e fasciculações em músculos da língua. Iniciouse tratamento com riluzole $100 \mathrm{mg} / \mathrm{dia}$, porém paciente não apresentou melhora, evoluindo para o óbito após três anos do início da doença.

Em nossos casos, o estudo da condução nervosa, bem como a investigação laboratorial e radiológica do encéfalo e da coluna cervical não evidenciaram alterações significativas, sendo o diagnóstico de ELA baseado nos critérios clínicos e neurofisiológicos nos casos 1 e 2; o caso 3 teve o diagnóstico de atrofia bulbar pro g ressiva. A Tabela 1 e a Figura mostram os achados da eletromiografia de agulha da região cervical e o aspecto clínico da paciente (Caso 2).

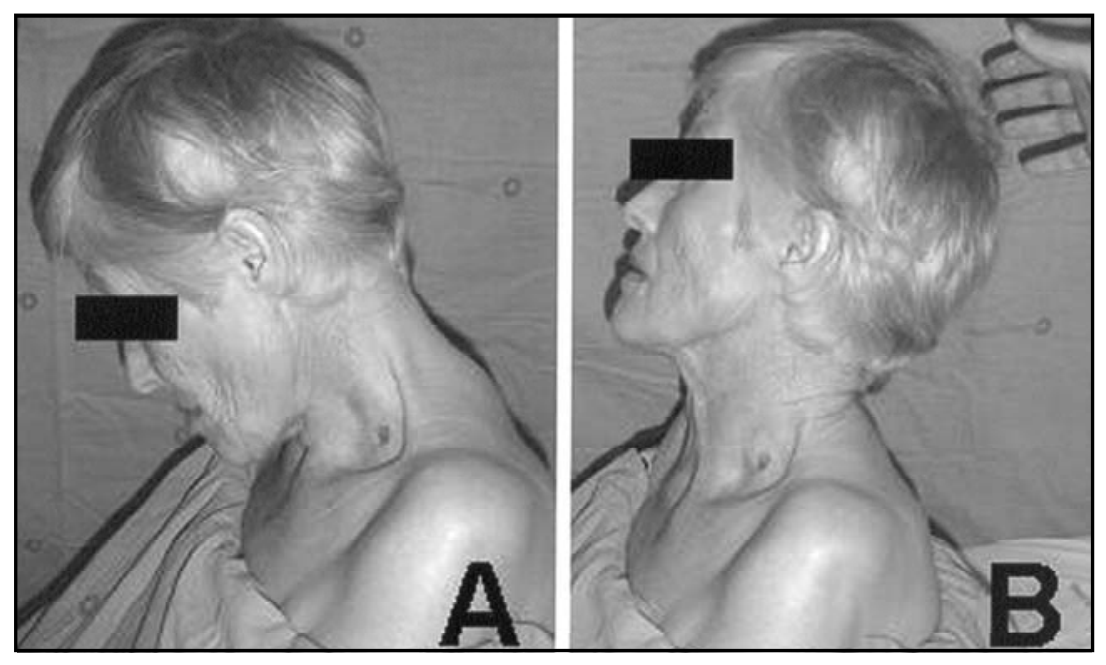

Figura. Aspecto clínico da SCC no caso 2. A) perfil demonstrando o aspecto do quei xo em contato com o a parede torácica e a cabeça caída. B) musculatura flexora cer vical pre s e rvada evitando a queda poste rior da cabeça (Publicação da foto autoriza da pelos familiares da paciente). 


\section{DISCUSSÃO}

Gourie-Devi et al. propuseram uma classificação etiológica para a SCC, dividindo os pacientes em quatro grupos conforme a doença a que estivessem relacionadas: miogênicas, neurogências, miscelânea e locais (Tabela 2) ${ }^{2}$. Dessa forma, os pacientes que apresentam SCC devem submeter-se a uma investigação etiológica extensa devido à possibilidade de doenças tratáveis como a miastenia gravis, miopatia inflamatória, entre outras, ou a miopatia cervical extensora isolada, as quais apresentam bom prognóstico. Dentre as causas de SCC, as DNM são pouco relatadas na literatura, sendo sua real prevalência difícil de ser estimada. Gourie-Devi et al. demonstraram a baixa freqüência desta manifestação em grupo de pacientes com ELA ao analisar 683 pacientes e encontrarem apenas $9(1,3 \%)$ com SCC 2 . Até o momento, há 15 casos descritos na literatura da associação entre SCC e ELA, e não encontramos descrição anterior de atrofia bulbar progressiva com SCC, mostrando a raridade desta

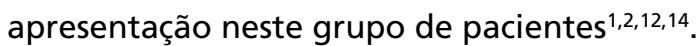

A SCC não foi descrita como manifestação inicial nas DNM, sendo encontrada apenas em pacientes com miopatia mitocondrial, miastenia gravis, hipopa$\mathrm{r}$ a t i reoidismo e hipotireoidismo ${ }^{3,9,10,15}$. As manifestações iniciais da DNM em nossas pacientes foram alterações bulbares, sendo caracterizadas por disfagia nos casos 1 e 3, e disartria no caso 2. Gourie-Devi et al. demonstraram apenas um paciente com manifestação bulbar inicial (disartria) e outros cinco pacientes com essa manifestação na pro g ressão da doença ${ }^{2}$. $O$ c o $\mathrm{m}$ p rometimento de musculatura de língua foi encontrado em todos os nossos casos, o que esta de a co rdocom os dados descritos em outros gru $\mathrm{pos}^{2}$. $\mathrm{O}$ acometimento da musculatura flexora cervical não foi encontrado em nenhum paciente do nosso relato.

Quando se relaciona o início da SCC ao tempo de evolução da ELA, podemos dividir os pacientes em dois grupos: precoce, com média de início entre 3 e 24 meses do diagnóstico de ELA; e tardio, entre 3 e 8 anos após o diagnóstico de ELA ${ }^{2}$. Todas as nossas pacientes apresentaram a SCC entre 1 mês e 2 anos após o início da doença, caracterizando o início precoce.

Os exames complementares necessários para a investigação da SCC estão relacionados à etiologia pesquisada; no entanto, a eletroneuromiografia é um dos principais exames por direcionar a investigação entre as doenças neurogênicas, miogênicas e de junção neuromuscular. Os achados eletroneuromiográ ficos em nossas pacientes foram compatíveis com o
Tabela 2. Classificação etiológica da síndrome da cabeça caída. (modificado de Gourie-Devi, et al. ${ }^{2}$ ).

Miogênica

Miastenia gravis

Polimiosite

Miopatia cervical extensora isolada

Distrofia fascioescapuloumeral

Miopatia nemalínica

Miopatia miotônica proximal

Miopatia por corpos de inclusão

Deficiência de carnitina

Deficiência da maltase ácida com início no adulto

Miopatia hipocalêmica aguda

Miopatia congênita

Miosite focal da musculatura extensora cervical

Miopatia mitocondrial

Neurogênica

Esclerose lateral amiotrófica

Atrofia muscular espinhal

Polineuropatia desmielinizante inflamatória crônica

Miscelânea

Hipotireoidismo

Distonia cervical

Parkinsonismo

Doença de Parkinson

Causas locais

Espondilite cervical com fraqueza neurogênica da musculatura extensora

Espondilite anquilosante

diagnóstico de DNM; alguns dos músculos extensores e flexores do pescoço mostraram sinais de desinervação ativa e crônica (Tabela 1). Na literatura, não encontramos descrição detalhada do padrão eletromiográfico da musculatura cervical em pacientes com ELA.

Os estudos de imagem por tomografia axial computadorizada e ressonância magnética de musculatura paraespinhal em miopatia cervical extensora isolada mostraram alterações semelhantes a edema e substituição gordurosa do músculo ${ }^{12,16}$. A descrição de atrofia e substituição gord u rosa da musculatura extensora cervical também foi observada em um paciente com miastenia gravis ${ }^{17}$. Em um caso associado a hipotireoidismo observou-se aumento do volume do músculo esplênio da cabeça bilateralmente 9 . $\mathrm{Na}$ DNM com SCC não encontramos estudos de imagem na literatura.

O tratamento da SCC na maioria dos casos deve ser relacionado à etiologia ${ }^{8,9}$. Nos pacientes com DNM ainda não há nenhum tratamento medicamentoso 
específico para SCC, mas ao identificar a DNM como etiologia da queda da cabeça, o uso de colar cervical deve ser estimulado, assim como a realização de fisioterapia, possibilitando melhora da postura².

\section{REFERÊNCIAS}

1. Lange DJ, Fetell MR, Lovelace RE, et al. The floppy head syndrome. Ann Neurol 1986;20:133.

2. Gourie-Devi M, Nalini A, Sandhya S. Early or late appearance of "d ropped head syndrome" in amyotrophic lateral sclerosis. J Neurol Neurosur Psychiatry 2003;74:683-686.

3. Puruckherr M, Pooyan P, Dube D, Byrd RP, Roy TM. The dropped head sign: an unusual presenting feature of myasthenia gravis. Neuromusc Disord 2004;14:378-379.

4. DeVere R, Bradley WG. Polymyositis: its presentation, morbidity and mortality. Brain 1975;98:637-666.

5. Padberg GW, Lunt PW, Koch M, et al. Diagnostic criteria for fascioscapulohumeral muscular dystrophy. Neuromusc Disord 1991;1:231-234.

6. Lomen-Hoerth C, Simmons ML, DeArmond SJ,et al. Adult-onset nemaline myopathy: another cause of dropped head. Muscle Nerve 1999; 22:1146-1150.

7. Karpati G, Carpenter S, Engel AG, et al. The syndrome of systemic carnitine deficiency: dinical, morphologic, biochemical and pathophysiology features. Neurology 1975;25:16-24.
8. Horiuchi M, Uehara Kenji, Kamo T, Sugihara H, Takahashi Y. Elderly onset Parkinson's disease complicated by dropped head syndrome that responded favorably to therapy. Geriatr Gerontol Internat 2004;4: 55-58.

9. Askmark H, Olsson Y, Rossitti S. Treatable dropped head syndrome in hypothyroidism. Neurology 2000;55:896-897.

10. Rahim F, Gupta D, Bertorini TE, LeDoux MS. Dropped head presentation of mitochondrial myopathy. J Clin Neuromusc Dis 2003;5:108-114.

11. Suare GA, Kelly JJ. The dropped head syndrome. Neurology 1992;42: 1625-1627.

12. Katz JS, Wolfe GI, Burns DK, Bryan WW, Fleckenstein JL, Barohn RJ. Isolated neck extensor myopathy: a common cause of dropped head syndrome. Neurology 1996;46:917-921.

13. Jaster JH, Bertorini TE, Swims MP, et al. Cervical kyphosis after resolution of myopathic head drop: a case report. Spine 1996;21:2023-2025.

14. Petiot P, Vial C, de Saint Victor JF, et al. Dropped head syndrome: diagnostic discussion apropos of 3 cases. Rev Neurol (Paris) 1997;153:251-255.

15. Beekman R, Tijssen CC, Visser LH, Schellens RLLA. Dropped head as the presenting symptom of primary hyperparathy roidism. J Neurol 2002;249:1738-1739.

16. Oerlemans WGH, de Visser M. Dropped head syndrome and bent spine syndrome: two separate clinical entities or different manifestations of axial myopathy? J Neurol Neurosurg Psychiatry 1998;65:258-259.

17. Rodolico C, Messina S, Toscano A, Vita G, Gaeta M. Axial myopathy in myasthenia: a misleading cause of dropped head. Muscle Nerve 2004;29:329-330. 